\title{
InteractE: Improving Convolution-Based Knowledge Graph Embeddings by Increasing Feature Interactions
}

\author{
Shikhar Vashishth, ${ }^{1 *}$ Soumya Sanyal, ${ }^{1 *}$ Vikram Nitin, ${ }^{2 \dagger}$ \\ Nilesh Agrawal, ${ }^{1}$ Partha Talukdar ${ }^{1}$ \\ ${ }^{1}$ Indian Institute of Science, ${ }^{2}$ Columbia University \\ \{shikhar, soumyasanyal, anilesh,ppt\}@iisc.ac.in \\ vikram.nitin@columbia.edu
}

\begin{abstract}
Most existing knowledge graphs suffer from incompleteness, which can be alleviated by inferring missing links based on known facts. One popular way to accomplish this is to generate low-dimensional embeddings of entities and relations, and use these to make inferences. ConvE, a recently proposed approach, applies convolutional filters on 2D reshapings of entity and relation embeddings in order to capture rich interactions between their components. However, the number of interactions that ConvE can capture is limited. In this paper, we analyze how increasing the number of these interactions affects link prediction performance, and utilize our observations to propose InteractE. InteractE is based on three key ideas - feature permutation, a novel feature reshaping, and circular convolution. Through extensive experiments, we find that InteractE outperforms state-of-the-art convolutional link prediction baselines on FB15k-237. Further, InteractE achieves an MRR score that is $9 \%, 7.5 \%$, and $23 \%$ better than ConvE on the FB15k-237, WN18RR and YAGO3-10 datasets respectively. The results validate our central hypothesis - that increasing feature interaction is beneficial to link prediction performance. We make the source code of InteractE available to encourage reproducible research.
\end{abstract}

\section{Introduction}

Knowledge graphs (KGs) are structured representations of facts, where nodes represent entities and edges represent relationships between them. This can be represented as a collection of triples $(s, r, o)$, each representing a relation $r$ between a "subject-entity" $s$ and an "object-entity" $o$. Some real-world knowledge graphs include Freebase (Bollacker et al. 2008), WordNet (Miller 1995), YAGO (Suchanek, Kasneci, and Weikum 2007), and NELL (Mitchell et al. 2018). KGs find application in a variety of tasks, such as relation extraction (Mintz et al. 2009; Vashishth et al. 2018), question answering (Bordes, Chopra, and Weston 2014), canonicalization (Vashishth, Jain, and Talukdar 2018), recommender systems (Zhang et al. 2016), and dialog systems (Ma et al. 2015).

\footnotetext{
* contributed equally to this paper.

${ }^{\dagger}$ Research done at Indian Institute of Science. Copyright (c) 2020, Association for the Advancement of Artificial Intelligence (www.aaai.org). All rights reserved.
}

However, most existing KGs are incomplete (Dong et al. 2014). The task of link prediction alleviates this drawback by inferring missing facts based on the known facts in a KG. A popular approach for solving this problem involves learning a low-dimensional representation for all entities and relations and utilizing them to predict new facts. In general, most existing link prediction methods learn to embed KGs by optimizing a score function which assigns higher scores to true facts than invalid ones. These score functions can be classified as translation distance based (Bordes et al. 2013; Xiao, Huang, and Zhu 2016; Wang et al. 2014a) or semantic matching based (Nickel, Rosasco, and Poggio 2016; Liu, Wu, and Yang 2017).

Recently, neural networks have also been utilized to learn the score function (Socher et al. 2013; Ravishankar, Chandrahas, and Talukdar 2017; Dettmers et al. 2018). The motivation behind these approaches is that shallow methods like TransE (Bordes et al. 2013) and DistMult (Yang et al. 2014) are limited in their expressiveness. As noted in (Dettmers et al. 2018), the only way to remedy this is to increase the size of their embeddings, which leads to an enormous increase in the number of parameters and hence limits their scalability to larger knowledge graphs.

Convolutional Neural Networks (CNN) have the advantage of using multiple layers, thus increasing their expressive power, while at the same time remaining parameterefficient. (Dettmers et al. 2018) exploit these properties and propose ConvE - a model which applies convolutional filters on stacked $2 \mathrm{D}$ reshapings of entity and relation embeddings. Through this, they aim to increase the number of interactions between components of these embeddings.

In this paper, we conclusively establish that increasing the number of such interactions is beneficial to link prediction performance, and show that the number of interactions that ConvE can capture is limited. We propose InteractE, a novel $\mathrm{CNN}$ based $\mathrm{KG}$ embedding approach which aims to further increase the interaction between relation and entity embeddings.

Our contributions are summarized as follows:

1. We propose InteractE, a method that augments the expressive power of ConvE through three key ideas - feature permutation, "checkered" feature reshaping, and cir- 


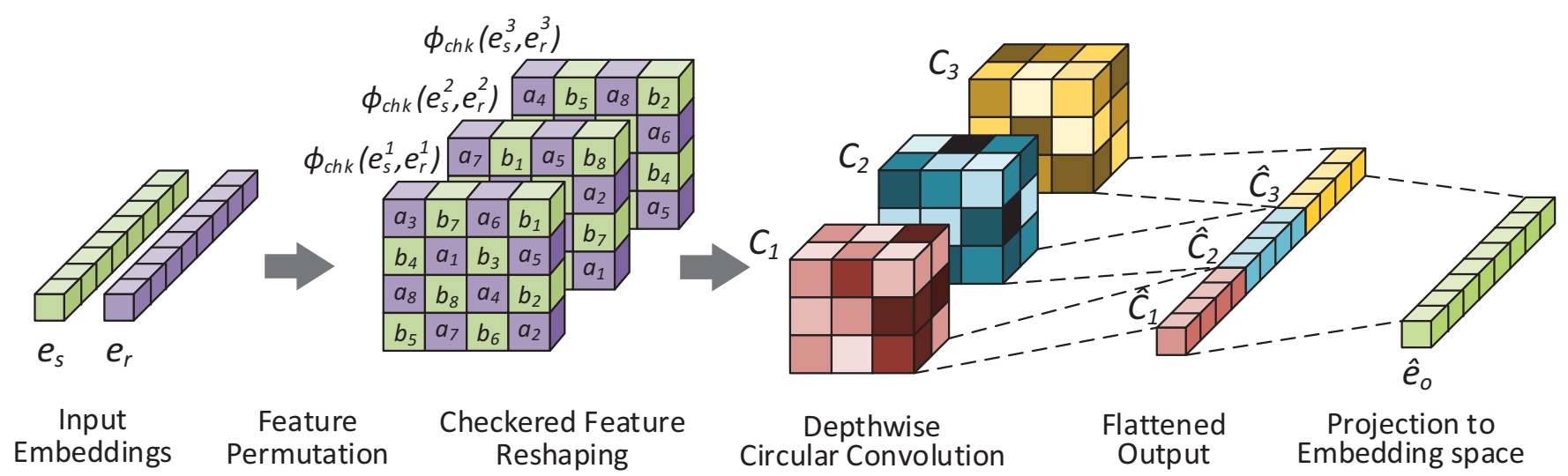

Figure 1: Overview of InteractE. Given entity and relation embeddings ( $\boldsymbol{e}_{s}$ and $\boldsymbol{e}_{r}$ respectively), InteractE generates multiple permutations of these embeddings and reshapes them using a "Checkered" reshaping function $\left(\phi_{\text {chk }}\right)$. Depth-wise circular convolution is employed to convolve each of the reshaped permutations $\left(C_{i}\right)$, which are then flattened $\left(\hat{C}_{i}\right)$ and fed to a fullyconnected layer to generate the predicted object embedding $\left(\widehat{e_{o}}\right)$. Please refer to Section 4 for details.

cular convolution.

2. We provide a precise definition of an interaction, and theoretically analyze InteractE to show that it increases interactions compared to ConvE. Further, we establish a correlation between the number of heterogeneous interactions (refer to Def. 3.2) and link prediction performance.

3. Through extensive evaluation on various link prediction datasets, we demonstrate InteractE's effectiveness (Section 8).

The source code of InteractE and datasets used in the paper have been made available at http://github.com/malllabiisc/ InteractE.

\section{Related Work}

Non-Neural: Starting with TransE (Bordes et al. 2013), there have been multiple proposed approaches that use simple operations like dot products and matrix multiplications to compute a score function. Most approaches embed entities as vectors, whereas for relations, vector (Bordes et al. 2013; Nickel, Rosasco, and Poggio 2016), matrix (Yang et al. 2014; Liu, Wu, and Yang 2017) and tensor (Lin et al. 2015) representations have been explored. For modeling uncertainty of learned representations, Gaussian distributions (He et al. 2015; Xiao, Huang, and Zhu 2016) have also been utilized. Methods like TransE (Bordes et al. 2013) and TransH (Wang et al. 2014a) utilize a translational objective for their score function, while DistMult (Yang et al. 2014) and ComplEx (Trouillon et al. 2016) use a bilinear diagonal based model.

Neural Network based: Recently, Neural Network (NN) based score functions have also been proposed. Neural Tensor Network (Socher et al. 2013) combines entity and relation embeddings by a relation-specific tensor which is given as input to a non-linear hidden layer for computing the score. (Dong et al. 2014; Ravishankar, Chandrahas, and Talukdar 2017) also utilize a Multi-Layer Perceptron for modeling the score function.

Convolution based: Convolutional Neural Networks (CNN) have also been employed for embedding Knowledge Graphs. ConvE (Dettmers et al. 2018) uses convolutional filters over reshaped subject and relation embeddings to compute an output vector and compares this with all other entities in the knowledge graph. Shang et al. propose ConvTransE a variant of the ConvE score function. They eschew $2 \mathrm{D}$ reshaping in favor of directly applying convolution on the stacked subject and relation embeddings. Further, they propose SACN which utilizes weighted graph convolution along with ConvTransE.

ConvKB (Nguyen et al. 2018) is another convolution based method which applies convolutional filters of width 1 on the stacked subject, relation and object embeddings for computing score. As noted in (Shang et al. 2019), although ConvKB was claimed to be superior to ConvE, its performance is not consistent across different datasets and metrics. Further, there have been concerns raised about the validity of its evaluation procedure (Sun et al. 2019b). Hence, we do not compare against it in this paper. A survey of all variants of existing KG embedding techniques can be found in (Nickel et al. 2016; Wang et al. 2017).

Most existing $\mathrm{KG}$ embedding approaches define an encoding for all entities and relations, i.e., $\boldsymbol{e}_{s}, \boldsymbol{e}_{r} \forall s \in \mathcal{E}, r \in$ $\mathcal{R}$. Then, a score function $\psi(s, r, o)$ is defined to measure the validity of triples. Table 1 lists some of the commonly used score functions. Finally, to learn the entity and relation representations, an optimization problem is solved for maximizing the plausibility of the triples $\mathcal{T}$ in the $\mathrm{KG}$.

ConvE: In this paper, we build upon ConvE (Dettmers et al. 2018), which models interaction between entities and relations using 2D Convolutional Neural Networks (CNN). The score function used is defined as follows:

$$
\psi(s, r, o)=f\left(\operatorname{vec}\left(f\left(\left[\overline{\boldsymbol{e}_{s}} ; \overline{\boldsymbol{e}_{r}} \star w\right]\right)\right) \boldsymbol{W}\right) \boldsymbol{e}_{\boldsymbol{o}},
$$

where, $\overline{\boldsymbol{e}_{\boldsymbol{s}}} \in \mathbb{R}^{d_{w} \times d_{h}}, \overline{\boldsymbol{e}_{\boldsymbol{r}}} \in \mathbb{R}^{d_{w} \times d_{h}}$ denote $2 \mathrm{D}$ reshapings of $\boldsymbol{e}_{\boldsymbol{s}} \in \mathbb{R}^{d_{w} d_{h} \times 1}, \boldsymbol{e}_{\boldsymbol{r}} \in \mathbb{R}^{d_{w} d_{h} \times 1}$, and $(\star)$ denotes the 


\begin{tabular}{lc}
\hline \multicolumn{1}{c}{ Model } & Scoring Function $\psi\left(\boldsymbol{e}_{s}, \boldsymbol{e}_{r}, \boldsymbol{e}_{o}\right)$ \\
\hline TransE & $\left\|\boldsymbol{e}_{s}+\boldsymbol{e}_{r}-\boldsymbol{e}_{o}\right\|_{p}$ \\
DistMult & $\left\langle\boldsymbol{e}_{s}, \boldsymbol{e}_{r}, \boldsymbol{e}_{o}\right\rangle$ \\
HolE & $\left\langle\boldsymbol{e}_{r}, \boldsymbol{e}_{s} * \boldsymbol{e}_{o}\right\rangle$ \\
ComplEx & $\operatorname{Re}\left(\left\langle\boldsymbol{e}_{s}, \boldsymbol{e}_{r}, \boldsymbol{e}_{o}\right\rangle\right)$ \\
ConvE & $f\left(\operatorname{vec}\left(f\left(\left[\boldsymbol{e}_{s} ; \overline{\boldsymbol{e}}_{r}\right] \star w\right)\right) \mathbf{W}\right) \boldsymbol{e}_{o}$ \\
RotatE & $-\left\|\boldsymbol{e}_{s} \circ \boldsymbol{e}_{r}-\boldsymbol{e}_{o}\right\|^{2}$ \\
\hline InteractE & $g\left(\operatorname{vec}\left(f\left(\phi\left(\mathcal{P}_{k}\right) \circledast w\right)\right) \boldsymbol{W}\right) \boldsymbol{e}_{o}$ \\
\hline
\end{tabular}

Table 1: The scoring functions $\psi(s, r, o)$ of various knowledge graph embedding methods. Here, $\boldsymbol{e}_{s}, \boldsymbol{e}_{r}, \boldsymbol{e}_{o} \in \mathbb{R}^{d}$ except for ComplEx and RotatE, where they are complex vectors $\left(\mathbb{C}^{d}\right), *$ denotes circular-correlation, $\star$ denotes convolution, $\circ$ represents Hadamard product and $\star$ denotes depthwise circular convolution operation.

convolution operation. The 2D reshaping enhances the interaction between entity and relation embeddings which has been found to be helpful for learning better representations (Nickel, Rosasco, and Poggio 2016).

\section{Notation and Definitions}

Let $\boldsymbol{e}_{s}=\left(a_{1}, \ldots, a_{d}\right), \boldsymbol{e}_{r}=\left(b_{1}, \ldots, b_{d}\right)$, where $a_{i}, b_{i} \in \mathbb{R} \forall i$, be an entity and a relation embedding respectively, and let $\boldsymbol{w} \in \mathbb{R}^{k \times k}$ be a convolutional kernel of size $k$. Further, we define that a matrix $M_{k} \in \mathbb{R}^{k \times k}$ is a $k$-submatrix of another matrix $N \in \mathbb{R}^{m \times n}$ if $\exists i, j$ such that $M_{k}=N_{i: i+k, j: j+k}$. We denote this by $M_{k} \subseteq N$.

Definition 3.1. (Reshaping Function) A reshaping function $\phi: \mathbb{R}^{d} \times \mathbb{R}^{d} \rightarrow \mathbb{R}^{m \times n}$ transforms embeddings $\boldsymbol{e}_{s}$ and $\boldsymbol{e}_{r}$ into a matrix $\phi\left(\boldsymbol{e}_{s}, \boldsymbol{e}_{r}\right)$, where $m \times n=2 d$. For conciseness, we abuse notation and represent $\phi\left(\boldsymbol{e}_{s}, \boldsymbol{e}_{r}\right)$ by $\phi$. We define three types of reshaping functions.

- Stack $\left(\phi_{s t k}\right)$ reshapes each of $\boldsymbol{e}_{s}$ and $\boldsymbol{e}_{r}$ into a matrix of shape $(m / 2) \times n$, and stacks them along their height to yield an $m \times n$ matrix (Fig. 2a). This is the reshaping function used in (Dettmers et al. 2018).

- Alternate $\left(\phi_{a l t}^{\tau}\right)$ reshapes $\boldsymbol{e}_{s}$ and $\boldsymbol{e}_{r}$ into matrices of shape $(m / 2) \times n$, and stacks $\tau$ rows of $\boldsymbol{e}_{s}$ and $\boldsymbol{e}_{r}$ alternately. In other words, as we decrease $\tau$, the "frequency" with which rows of $\boldsymbol{e}_{s}$ and $\boldsymbol{e}_{r}$ alternate increases. We denote $\phi_{\text {alt }}^{1}(\tau=1)$ as $\phi_{\text {alt }}$ for brevity (Fig. 2b).

- Chequer $\left(\phi_{c h k}\right)$ arranges $\boldsymbol{e}_{s}$ and $\boldsymbol{e}_{r}$ such that no two adjacent cells are occupied by components of the same embedding (Fig. 2c).

Definition 3.2. (Interaction) An interaction is defined as a triple $\left(x, y, M_{k}\right)$, such that $M_{k} \subseteq \phi\left(\boldsymbol{e}_{s}, \boldsymbol{e}_{r}\right)$ is a $k$-submatrix of the reshaped input embeddings; $x, y \in M_{k}$ and are distinct components of $\boldsymbol{e}_{s}$ or $\boldsymbol{e}_{r}$. The number of interactions $\mathcal{N}(\phi, k)$ is defined as the cardinality of the set of all possible triples. Note that $\phi$ can be replaced with $\Omega(\phi)$ for some padding function $\Omega$.

An interaction $\left(x, y, M_{k}\right)$ is called heterogeneous if $x$ and $y$ are components of $\boldsymbol{e}_{s}$ and $\boldsymbol{e}_{r}$ respectively, or vice-

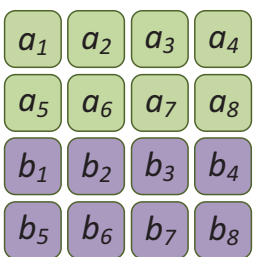

(a) Stack

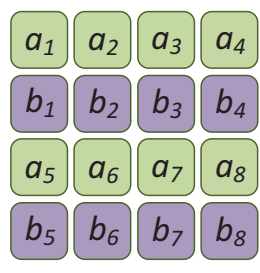

(b) Alternate (c) Chequer

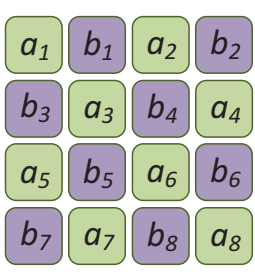

Figure 2: Different types of reshaping functions we analyze in this paper. Here, $\boldsymbol{e}_{s}=\left(a_{1}, \ldots, a_{8}\right), \boldsymbol{e}_{r}=\left(b_{1}, \ldots, b_{8}\right)$, and $m=n=4$. Please refer to Section 3 for more details.

versa. Otherwise, it is called homogeneous. We denote the number of heterogeneous and homogeneous interactions as $\mathcal{N}_{\text {het }}(\phi, k)$ and $\mathcal{N}_{\text {homo }}(\phi, k)$ respectively. For example, in a $3 \times 3$ matrix $M_{3}$, if there are 5 components of $\boldsymbol{e}_{s}$ and 4 of $\boldsymbol{e}_{r}$, then the number of heterogeneous and homogeneous interactions are: $\mathcal{N}_{\text {het }}=2(5 \times 4)=40$, and $\mathcal{N}_{\text {homo }}=2\left[\left(\begin{array}{l}5 \\ 2\end{array}\right)+\left(\begin{array}{l}4 \\ 2\end{array}\right)\right]=32$. Please note that the sum of total number of heterogenous and homogenous interactions in a reshaping function is constant and is equal to $2\left(\begin{array}{c}k^{2} \\ 2\end{array}\right)$, i.e., $\mathcal{N}_{\text {het }}(\phi, k)+\mathcal{N}_{\text {homo }}(\phi, k)=2\left(\begin{array}{c}k^{2} \\ 2\end{array}\right)$.

\section{InteractE Overview}

Recent methods (Yang et al. 2014; Nickel, Rosasco, and Poggio 2016) have demonstrated that expressiveness of a model can be enhanced by increasing the possible interactions between embeddings. ConvE (Dettmers et al. 2018) also exploits the same principle albeit in a limited way, using convolution on $2 \mathrm{D}$ reshaped embeddings. InteractE extends this notion of capturing entity and relation feature interactions using the following three ideas:

- Feature Permutation: Instead of using one fixed order of the input, we utilize multiple permutations to capture more possible interactions.

- Checkered Reshaping: We substitute simple feature reshaping of ConvE with checked reshaping and prove its superiority over other possibilities.

- Circular Convolution: Compared to the standard convolution, circular convolution allows to capture more feature interactions as depicted in Figure 3. The convolution is performed in a depth-wise manner (Chollet 2017) on different input permutations.

\section{InteractE Details}

In this section, we provide a detailed description of the various components of InteractE. The overall architecture is depicted in Fig. 1. InteractE learns a $d$-dimensional vector representation $\left(\boldsymbol{e}_{s}, \boldsymbol{e}_{r} \in \mathbb{R}^{d}\right)$ for each entity and relation in the knowledge graph, where $d=d_{w} d_{h}$.

\subsection{Feature Permutation}

To capture a variety of heterogeneous interactions, InteractE first generates $t$-random permutations of both $e_{s}$ and $e_{r}$, de- 
noted by $\mathcal{P}_{t}=\left[\left(\boldsymbol{e}_{s}^{1}, \boldsymbol{e}_{r}^{1}\right) ; \ldots ;\left(\boldsymbol{e}_{s}^{t}, \boldsymbol{e}_{r}^{t}\right)\right]$. Note that with high probability, the sets of interactions within $\phi\left(\boldsymbol{e}_{s}^{i}, \boldsymbol{e}_{r}^{i}\right)$ for different $i$ are disjoint. This is evident because the number of distinct interactions across all possible permutations is very large. So, for $t$ different permutations, we can expect the total number of interactions to be approximately $t$ times the number of interactions for one permutation.

\subsection{Checkered Reshaping}

Next, we apply the reshaping operation $\phi_{c h k}\left(\boldsymbol{e}_{s}^{i}, \boldsymbol{e}_{r}^{i}\right), \forall i \in$ $\{1, \ldots, t\}$, and define $\phi\left(\mathcal{P}_{t}\right)=\left[\phi\left(\boldsymbol{e}_{s}^{1}, \boldsymbol{e}_{r}^{1}\right) ; \ldots ; \phi\left(\boldsymbol{e}_{s}^{t}, \boldsymbol{e}_{r}^{t}\right)\right]$. ConvE (Dettmers et al. 2018) uses $\phi_{s t k}(\cdot)$ as a reshaping function which has limited interaction capturing ability. On the basis of Proposition 6.3, we choose to utilize $\phi_{c h k}(\cdot)$ as the reshaping function in InteractE, which captures maximum heterogeneous interactions between entity and relation features.

\subsection{Circular Convolution}

Motivated by our analysis in Proposition 6.4, InteractE uses circular convolution, which further increases interactions compared to the standard convolution. This has been successfully applied for tasks like image recognition (Wang et al. 2018). Circular convolution on a 2-dimensional input $\boldsymbol{I} \in \mathbb{R}^{m \times n}$ with a filter $w \in \mathbb{R}^{k \times k}$ is defined as:

$$
[\boldsymbol{I} \star \boldsymbol{w}]_{p, q}=\sum_{i=-\lfloor k / 2\rfloor}^{\lfloor k / 2\rfloor} \sum_{j=-\lfloor k / 2\rfloor}^{\lfloor k / 2\rfloor} \boldsymbol{I}_{[p-i]_{m},[q-j]_{n}} \boldsymbol{w}_{i, j},
$$

where, $[x]_{n}$ denotes $x$ modulo $n$ and $\lfloor\cdot\rfloor$ denotes the floor function. Figure 3 and Proposition 6.4 show how circular convolution captures more interactions compared to standard convolution with zero padding.

InteractE stacks each reshaped permutation as a separate channel. For convolving permutations, we apply circular convolution in a depth-wise manner (Chollet 2017). Although different sets of filters can be applied for each permutation, in practice we find that sharing filters across channels works better as it allows a single set of kernel weights to be trained on more input instances.

\subsection{Score Function}

The output of each circular convolution is flattened and concatenated into a vector. InteractE then projects this vector to the embedding space $\left(\mathbb{R}^{d}\right)$. Formally, the score function used in InteractE is defined as follows:

$$
\psi(s, r, o)=g\left(\operatorname{vec}\left(f\left(\phi\left(\boldsymbol{P}_{k}\right) \circledast \boldsymbol{w}\right)\right) \boldsymbol{W}\right) \boldsymbol{e}_{o},
$$

where $\circledast$ denotes depth-wise circular convolution, $\operatorname{vec}(\cdot)$ denotes vector concatenation, $\boldsymbol{e}_{o}$ represents the object entity embedding matrix and $W$ is a learnable weight matrix. Functions $f$ and $g$ are chosen to be ReLU and sigmoid respectively. For training, we use the standard binary cross entropy loss with label smoothing.

\section{Theoretical Analysis}

In this section, we analyze multiple variants of 2D reshaping with respect to the number of interactions they induce. We

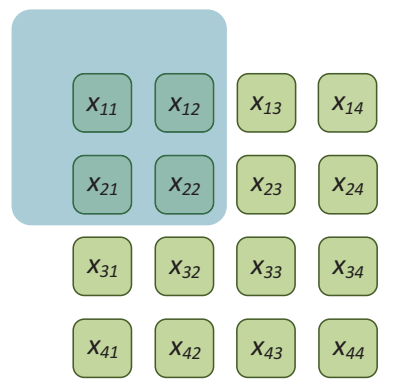

(a) Standard Convolution

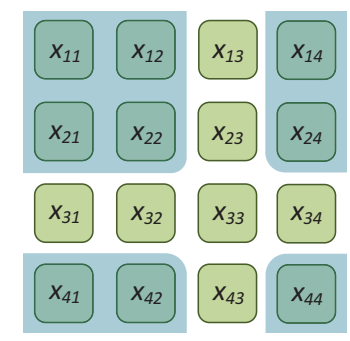

(b) Circular Convolution

Figure 3: Circular convolution induces more interactions than standard convolution. Here, $X$ is a $4 \times 4$ input matrix with components $x_{i j}$. The shaded region depicts where the filter is applied. Please refer to Section 5.3 for more details.

also examine the advantages of using circular padded convolution over the standard convolution.

For simplicity, we restrict our analysis to the case where the output of the reshaping function is a square matrix, i.e., $m=n$. Note that our results can be extended to the general case as well. Proofs of all propositions herein are included in the supplementary material.

Proposition 6.1. For any kernel $\boldsymbol{w}$ of size $k$, for all $n \geq$ $\left(\frac{5 k}{3}-1\right)$ if $k$ is odd and $n \geq \frac{(5 k+2)(k-1)}{3 k}$ if $k$ is even, the following statement holds:

$$
\mathcal{N}_{\text {het }}\left(\phi_{\text {alt }}, k\right) \geq \mathcal{N}_{\text {het }}\left(\phi_{\text {st }}, k\right)
$$

Proposition 6.2. For any kernel $\boldsymbol{w}$ of size $k$ and for all $\tau<$ $\tau^{\prime}\left(\tau, \tau^{\prime} \in \mathbb{N}\right)$, the following statement holds:

$$
\mathcal{N}_{\text {het }}\left(\phi_{\text {alt }}^{\tau}, k\right) \geq \mathcal{N}_{\text {het }}\left(\phi_{\text {alt }}^{\tau^{\prime}}, k\right)
$$

Proposition 6.3. For any kernel $\boldsymbol{w}$ of size $k$ and for all reshaping functions $\phi: \mathbb{R}^{d} \times \mathbb{R}^{d} \rightarrow \mathbb{R}^{n \times n}$, the following statement holds:

$$
\mathcal{N}_{\text {het }}\left(\phi_{\text {chk }}, k\right) \geq \mathcal{N}_{\text {het }}(\phi, k)
$$

Proposition 6.4. Let $\Omega_{0}, \Omega_{c}: \mathbb{R}^{n \times n} \rightarrow \mathbb{R}^{(n+p) \times(n+p)}$ denote zero padding and circular padding functions respectively, for some $p>0$. Then for any reshaping function $\phi$,

$$
\mathcal{N}_{\text {het }}\left(\Omega_{c}(\phi), k\right) \geq \mathcal{N}_{\text {het }}\left(\Omega_{0}(\phi), k\right)
$$

\section{Experimental Setup}

\subsection{Datasets}

In our experiments, following (Dettmers et al. 2018; Sun et al. 2019a), we evaluate on the three most commonly used link prediction datasets. A summary statistics of the datasets is presented in Table 3 .

- FB15k-237 (Toutanova and Chen 2015) is a improved version of FB15k (Bordes et al. 2013) dataset where all inverse relations are deleted to prevent direct inference of test triples by reversing training triples. 


\begin{tabular}{|c|c|c|c|c|c|c|c|c|c|c|c|c|}
\hline & \multicolumn{4}{|c|}{ FB15k-237 } & \multicolumn{4}{|c|}{ WN18RR } & \multicolumn{4}{|c|}{ YAGO3-10 } \\
\hline & MRR & MR & H@ 10 & H@1 & MRR & MR & H@ 10 & H@1 & MRR & MR & H@ 10 & H@1 \\
\hline DistMult (Yang et al. 2014) & .241 & 254 & .419 & .155 & .430 & 5110 & .49 & .39 & .34 & 5926 & .54 & .24 \\
\hline ComplEx (Trouillon et al. 2016) & .247 & 339 & .428 & .158 & .44 & 5261 & .51 & .41 & .36 & 6351 & .55 & 26 \\
\hline R-GCN (Schlichtkrull et al. 2017) & .248 & - & .417 & .151 & - & - & - & -7 & - & - & - & - \\
\hline KBGAN (Cai and Wang 2018) & .278 & - & .458 & - & .214 & - & .472 & - & - & - & - & - \\
\hline KBLRN (García-Durán and Niepert 2018) & .309 & 209 & .493 & .219 & - & - & - & - & - & - & - & - \\
\hline ConvTransE (Shang et al. 2019) & .33 & - & .51 & .24 & .46 & - & .52 & .43 & - & - & - & - \\
\hline SACN (Shang et al. 2019) & .35 & - & .54 & .26 & .47 & - & .54 & .43 & - & - & - & - \\
\hline RotatE (Sun et al. 2019a) & .338 & 177 & .533 & .241 & .476 & 3340 & .571 & .428 & .495 & 1767 & .670 & .402 \\
\hline ConvE (Dettmers et al. 2018) & .325 & 244 & .501 & .237 & .43 & 4187 & .52 & .40 & .44 & 1671 & .62 & .35 \\
\hline InteractE (Proposed Method) & .354 & 172 & .535 & .263 & .463 & 5202 & .528 & .430 & .541 & 2375 & .687 & .462 \\
\hline
\end{tabular}

Table 2: Link prediction results of several models evaluated on FB15k-237, WN18RR and YAGO3-10. We find that InteractE outperforms all other methods across metrics on FB15k-237 and in 3 out of 4 settings on YAGO3-10. Since InteractE generalizes ConvE, we highlight performance comparison between the two methods specifically in the table above. Please refer to Section 8.1 for more details.

- WN18RR (Dettmers et al. 2018) is a subset of WN18 (Bordes et al. 2013) derived from WordNet (Miller 1995), with deleted inverse relations similar to FB15k-237.

- YAGO3-10 is a subset of YAGO3 (Suchanek, Kasneci, and Weikum 2007) constitutes entities with at least 10 relations. Triples consist of descriptive attributes of people.

\subsection{Evaluation protocol}

Following (Bordes et al. 2013), we use the filtered setting, i.e., while evaluating on test triples, we filter out all the valid triples from the candidate set, which is generated by either corrupting the head or tail entity of a triple. The performance is reported on the standard evaluation metrics: Mean Reciprocal Rank (MRR), Mean Rank (MR) and Hits@1, and Hits@10. We report average results across 5 runs. We note that the variance is substantially low on all the metrics and hence omit it.

\subsection{Baselines}

In our experiments, we compare InteractE against a variety of baselines which can be categorized as:

- Non-neural: Methods that use simple vector based operations for computing score. For instance, DistMult (Yang et al. 2014), ComplEx (Trouillon et al. 2016), KBGAN (Cai and Wang 2018), KBLRN (García-Durán and Niepert 2018) and RotatE (Sun et al. 2019a).

- Neural: Methods which leverage a non-linear neural network based architecture in their scoring function. This includes R-GCN (Schlichtkrull et al. 2017), ConvE (Dettmers et al. 2018), ConvTransE (Shang et al. 2019), and SACN (Shang et al. 2019).

\section{Results}

In this section, we attempt to answer the questions below:

Q1. How does InteractE perform in comparison to the existing approaches? (Section 8.1)

\begin{tabular}{lrrrrr}
\hline Dataset & $|\mathcal{E}|$ & $|\mathcal{R}|$ & \multicolumn{3}{c}{ \# Triples } \\
\cline { 3 - 6 } & & & \multicolumn{1}{c}{ Train } & Valid & \multicolumn{1}{c}{ Test } \\
\hline FB15k-237 & 14,541 & 237 & 272,115 & 17,535 & 20,466 \\
WN18RR & 40,943 & 11 & 86,835 & 3,034 & 3,134 \\
YAGO3-10 & 123,182 & 37 & $1,079,040$ & 5,000 & 5,000 \\
\hline
\end{tabular}

Table 3: Details of the datasets used. Please see Section 7.1 for more details.

Q2. What is the effect of different feature reshaping and circular convolution on link prediction performance? (Section 8.2)

Q3. How does the performace of our model vary with number of feature permutations? (Section 8.3)

Q4. What is the performance of InteractE on different relation types? (Section 8.4)

\subsection{Performance Comparison}

In order to evaluate the effectiveness of InteractE, we compare it against the existing knowledge graph embedding methods listed in Section 7.3. The results on three standard link prediction datasets are summarized in Table 2. The scores of all the baselines are taken directly from the values reported in the papers (Dettmers et al. 2018; Sun et al. 2019a; Shang et al. 2019; Cai and Wang 2018; García-Durán and Niepert 2018). Since our model builds on ConvE, we specifically compare against it, and find that InteractE outperforms ConvE on all metrics for FB15k237 and WN18RR and on three out of four metrics on YAGO3-10. On an average, InteractE obtains an improvement of $9 \%, 7.5 \%$, and $23 \%$ on FB15k-237, WN18RR, and YAGO3-10 on MRR over ConvE. This validates our hypothesis that increasing heterogeneous interactions help improve performance on link prediction. For YAGO3-10, we observe that the MR obtained from InteractE is worse than ConvE although it outperforms ConvE on all other metrics. Simliar trend has been observed in (Dettmers et al. 2018; Sun et al. 2019a). 


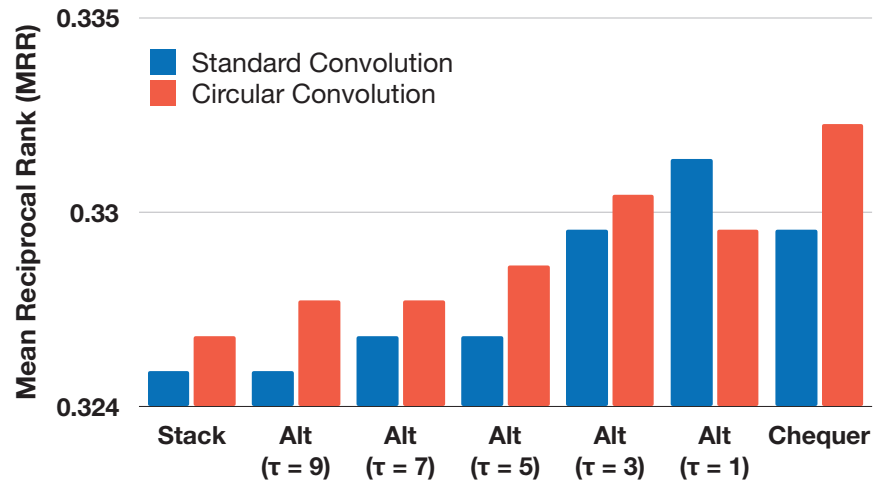

(a) FB15k-237 dataset

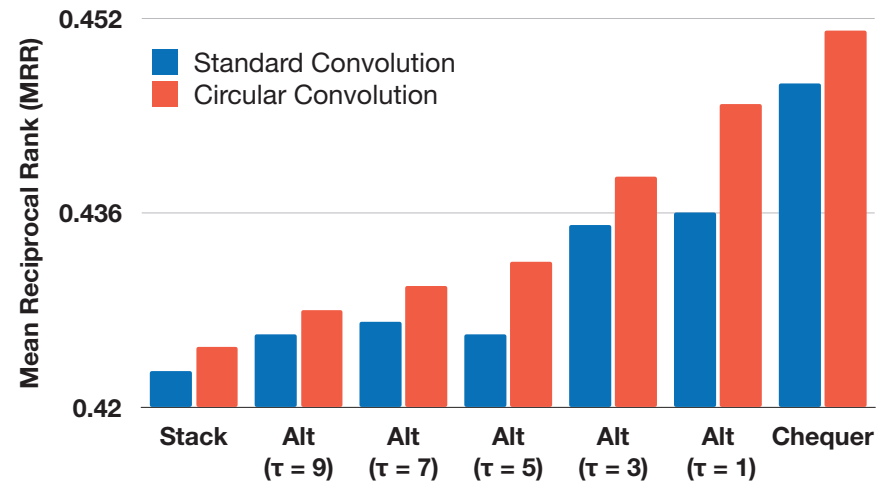

(b) WN18RR dataset

Figure 4: Performance with different feature reshaping and convolution operation on validation data of FB15k-237 and WN18RR. Stack and Alt denote Stacked and Alternate reshaping as defined in Section 3. As we decrease $\tau$ the number of heterogeneous interactions increases (refer to Proposition 6.2). The results empirically verify our theoretical claim in Section 6 and validate the central thesis of this paper that increasing heterogeneous interactions improves link prediction performance. Please refer to Section 8.2 for more details.

Compared to other baseline methods, InteractE outperforms them on FB15k-237 across all the metrics and on 3 out of 4 metrics on YAGO3-10 dataset. The below-par performance of InteractE on WN18RR can be attributed to the fact that this dataset is more suitable for shallow models as it has very low average relation-specific in-degree. This is consistent with the observations of (Dettmers et al. 2018).

\subsection{Effect of Feature Reshaping and Circular Convolution}

In this section, we empirically test the effectiveness of different reshaping techniques we analyzed in Section 6. For this, we evaluate different variants of InteractE on validation data of FB15k-237 and WN18RR with the number of feature permutations set to 1 . We omit the analysis on YAGO310 given its large size. The results are summarized in Figure 4. We find that the performance with Stacked reshaping is the worst, and it improves when we replace it with alternate reshaping. This observation is consistent with our findings in Proposition 6.1. Further, we find that MRR improves on decreasing the value of $\tau$ in alternate reshaping, which empirically validates Proposition 6.2. Finally, we observe that checkered reshaping gives the best performance across all reshaping functions for most scenarios, thus justifying Proposition 6.3.

We also compare the impact of using circular and standard convolution on link prediction performance. The MRR scores are reported in Figure 4. The results show that circular convolution is consistently better than the standard convolution. This also verifies our statement in Proposition 6.4. Overall, we find that increasing interaction helps improve performance on the link prediction task, thus validating the central thesis of our paper.

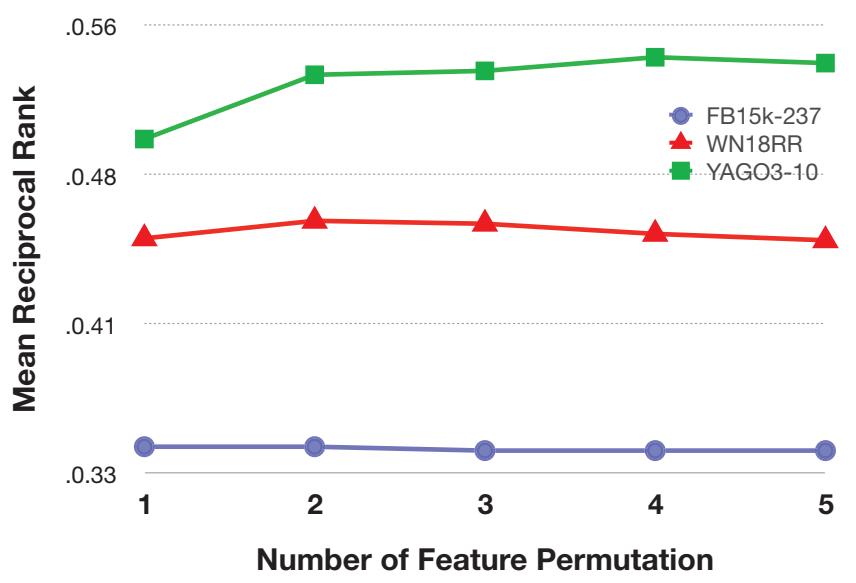

Figure 5: Performance on the validation data of FB15k-237, WN18RR, and YAGO3-10 with different numbers of feature permutations. We find that although increasing the number of permutations improves performance, it saturates as we exceed a certain limit. Please see Section 8.3 for details.

\subsection{Effect of Feature Permutations}

In this section, we analyze the effect of increasing the number of feature permutations on InteractE's performance on validation data of FB15k-237, WN18RR, and YAGO3-10. The overall results are summarized in Figure 5. We observe that on increasing the number of permuations although on FB15k-237, MRR remains the same, it improves on WN18RR and YAGO3-10 datasets. However, it degrades as the number of permutations is increased beyond a certain limit. We hypothesize that this is due to overparameteralization of the model. Moreover, since the number of relevant interactions are finite, increasing the number of permutations could become redundant beyond a limit. 


\begin{tabular}{|c|c|c|c|c|c|c|c|c|c|c|}
\hline & & \multicolumn{3}{|c|}{ RotatE } & \multicolumn{3}{|c|}{ ConvE } & \multicolumn{3}{|c|}{ InteractE } \\
\hline & & MRR & MR & H@10 & MRR & MR & H@10 & MRR & MR & H@ 10 \\
\hline \multirow{4}{*}{ 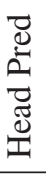 } & $1-1$ & 0.498 & 359 & 0.593 & 0.374 & 223 & 0.505 & 0.386 & 175 & 0.547 \\
\hline & $1-\mathrm{N}$ & 0.092 & 614 & 0.174 & 0.091 & 700 & 0.17 & 0.106 & 573 & 0.192 \\
\hline & $\mathrm{N}-1$ & 0.471 & 108 & 0.674 & 0.444 & 73 & 0.644 & 0.466 & 69 & 0.647 \\
\hline & $\mathrm{N}-\mathrm{N}$ & 0.261 & 141 & 0.476 & 0.261 & 158 & 0.459 & 0.276 & 148 & 0.476 \\
\hline \multirow{4}{*}{ 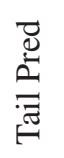 } & $1-1$ & 0.484 & 307 & 0.578 & 0.366 & 261 & 0.51 & 0.368 & 308 & 0.547 \\
\hline & $1-\mathrm{N}$ & 0.749 & 41 & 0.674 & 0.762 & 33 & 0.878 & 0.777 & 27 & 0.881 \\
\hline & $\mathrm{N}-1$ & 0.074 & 578 & 0.138 & 0.069 & 682 & 0.15 & 0.074 & 625 & 0.141 \\
\hline & $\mathrm{N}-\mathrm{N}$ & 0.364 & 90 & 0.608 & 0.375 & 100 & 0.603 & 0.395 & 92 & 0.617 \\
\hline
\end{tabular}

Table 4: Link prediction results by relation category on FB15k-237 dataset for RotatE, ConvE, and InteractE. Following (Wang et al., 2014b), the relations are categorized into one-to-one (1-1), one-to-many (1-N), many-to-one (N-1), and many-to-many $(\mathrm{N}-\mathrm{N})$. We observe that InteractE is effective at capturing complex relations compared to RotatE. Refer to Section 8.4 for details.

\subsection{Evaluation on different Relation Types}

In this section, we analyze the performance of InteractE on different relation categories of FB15k-237. We chose FB15k-237 for analysis over other datasets because of its more and diverse set of relations. Following (Wang et al. $2014 b$ ), we classify the relations based on the average number of tails per head and heads per tail into four categories: one-to-one, one-to-many, many-to-one, and many-to-many. The results are presented in Table 4. Overall, we find that InteractE is effective at modeling complex relation types like one-to-many and many-to-many whereas, RotatE captures simple relations like one-to-one better. This demonstrates that an increase in interaction allows the model to capture more complex relationships.

\section{Conclusion}

In this paper, we propose InteractE, a novel knowledge graph embedding method which alleviates the limitations of ConvE by capturing additional heterogeneous feature interactions. InteractE is able to achieve this by utilizing three central ideas, namely feature permutation, checkered feature reshaping, and circular convolution. Through extensive experiments, we demonstrate that InteractE achieves a consistent improvement on link prediction performance on multiple datasets. We also theoretically analyze the effectiveness of the components of InteractE, and provide empirical validation of our hypothesis that increasing heterogeneous feature interaction is beneficial for link prediction with ConvE. This work demonstrates a possible scope for improving existing knowledge graph embedding methods by leveraging rich heterogenous interactions.

\section{Acknowledgments}

We thank the anonymous reviewers for their constructive comments. This work is supported in part by the Ministry of Human Resource Development (Government of India) and Google PhD Fellowship.

\section{References}

Bollacker, K.; Evans, C.; Paritosh, P.; Sturge, T.; and Taylor, J. 2008. Freebase: A collaboratively created graph database for structuring human knowledge. In Proceedings of the 2008 ACM SIGMOD International Conference on Management of Data, SIGMOD '08, 1247-1250. New York, NY, USA: ACM.

Bordes, A.; Usunier, N.; Garcia-Duran, A.; Weston, J.; and Yakhnenko, O. 2013. Translating embeddings for modeling multirelational data. In Advances in Neural Information Processing Systems. 2787-2795.

Bordes, A.; Chopra, S.; and Weston, J. 2014. Question answering with subgraph embeddings. In Proceedings of the 2014 Conference on Empirical Methods in Natural Language Processing, 615-620.

Cai, L., and Wang, W. Y. 2018. KBGAN: Adversarial learning for knowledge graph embeddings. In Proceedings of the 2018 Conference of the North American Chapter of the Association for Computational Linguistics: Human Language Technologies, 1470-1480.

Chollet, F. 2017. Xception: Deep learning with depthwise separable convolutions. In Proceedings of Conference on Computer Vision and Pattern Recognition.

Dettmers, T.; Pasquale, M.; Pontus, S.; and Riedel, S. 2018. Convolutional $2 \mathrm{~d}$ knowledge graph embeddings. In Proceedings of the 32th AAAI Conference on Artificial Intelligence, 1811-1818.

Dong, X.; Gabrilovich, E.; Heitz, G.; Horn, W.; Lao, N.; Murphy, K.; Strohmann, T.; Sun, S.; and Zhang, W. 2014. Knowledge vault: A web-scale approach to probabilistic knowledge fusion. In International Conference on Knowledge Discovery and Data Mining, 601-610.

García-Durán, A., and Niepert, M. 2018. Kblrn: End-to-end learning of knowledge base representations with latent, relational, and numerical features. In Proceedings of the Thirty-Fourth Conference on Uncertainty in Artificial Intelligence, UAI 2018, Monterey, California, USA, August 6-10, 2018, 372-381.

He, S.; Liu, K.; Ji, G.; and Zhao, J. 2015. Learning to represent knowledge graphs with gaussian embedding. In Proceedings of the 24th ACM International on Conference on Information and Knowledge Management, CIKM '15, 623-632. New York, NY, USA: ACM.

Lin, Y.; Liu, Z.; Sun, M.; Liu, Y.; and Zhu, X. 2015. Learning entity and relation embeddings for knowledge graph completion. In Proceedings of the Twenty-Ninth AAAI Conference on Artificial Intelligence, AAAI'15, 2181-2187. AAAI Press. 
Liu, H.; Wu, Y.; and Yang, Y. 2017. Analogical inference for multi-relational embeddings. In Precup, D., and Teh, Y. W., eds., 34th International Conference on Machine Learning, volume 70, 2168-2178.

Ma, Y.; Crook, P. A.; Sarikaya, R.; and Fosler-Lussier, E. 2015. Knowledge graph inference for spoken dialog systems. In 2015 IEEE International Conference on Acoustics, Speech and Signal Processing.

Miller, G. A. 1995. Wordnet: A lexical database for english. Commun. ACM 38(11):39-41.

Mintz, M.; Bills, S.; Snow, R.; and Jurafsky, D. 2009. Distant supervision for relation extraction without labeled data. In Proceedings of the 47th Annual Meeting of the ACL, 1003-1011.

Mitchell, T.; Cohen, W.; Hruschka, E.; Talukdar, P.; Yang, B.; Betteridge, J.; Carlson, A.; Dalvi, B.; Gardner, M.; Kisiel, B.; Krishnamurthy, J.; Lao, N.; Mazaitis, K.; Mohamed, T.; Nakashole, N.; Platanios, E.; Ritter, A.; Samadi, M.; Settles, B.; Wang, R.; Wijaya, D.; Gupta, A.; Chen, X.; Saparov, A.; Greaves, M.; and Welling, J. 2018. Never-ending learning. Commun. ACM 103-115.

Nguyen, D. Q.; Nguyen, T. D.; Nguyen, D. Q.; and Phung, D. 2018. A novel embedding model for knowledge base completion based on convolutional neural network. In Proceedings of North American Chapter of the Association for Computational Linguistics, 327-333.

Nickel, M.; Murphy, K.; Tresp, V.; and Gabrilovich, E. 2016. A review of relational machine learning for knowledge graphs. Proceedings of the IEEE 104(1):11-33.

Nickel, M.; Rosasco, L.; and Poggio, T. 2016. Holographic embeddings of knowledge graphs. In Proceedings of the Thirtieth Conference on Artificial Intelligence, AAAI'16, 1955-1961.

Ravishankar, S.; Chandrahas; and Talukdar, P. P. 2017. Revisiting simple neural networks for learning representations of knowledge graphs. CoRR abs/1711.05401.

Schlichtkrull, M.; Kipf, T. N.; Bloem, P.; Berg, R. v. d.; Titov, I.; and Welling, M. 2017. Modeling relational data with graph convolutional networks. arXiv preprint arXiv:1703.06103.

Shang, C.; Tang, Y.; Huang, J.; Bi, J.; He, X.; and Zhou, B. 2019. End-to-end structure-aware convolutional networks for knowledge base completion. In Proceedings of the AAAI Conference on Artificial Intelligence, volume 33, 3060-3067.

Socher, R.; Chen, D.; Manning, C. D.; and Ng, A. Y. 2013. Reasoning with neural tensor networks for knowledge base completion. In Proceedings of the 26th International Conference on Neural Information Processing Systems, NIPS'13, 926-934.

Suchanek, F. M.; Kasneci, G.; and Weikum, G. 2007. Yago: A Core of Semantic Knowledge. In 16th International Conference on the World Wide Web, 697-706.

Sun, Z.; Deng, Z.-H.; Nie, J.-Y.; and Tang, J. 2019a. Rotate: Knowledge graph embedding by relational rotation in complex space. In International Conference on Learning Representations.

Sun, Z.; Vashishth, S.; Sanyal, S.; Talukdar, P.; and Yang, Y. 2019b. A Re-evaluation of Knowledge Graph Completion Methods. arXiv e-prints arXiv:1911.03903.

Toutanova, K., and Chen, D. 2015. Observed versus latent features for knowledge base and text inference. In Proceedings of the $3 \mathrm{rd}$ Workshop on Continuous Vector Space Models, 57-66.

Trouillon, T.; Welbl, J.; Riedel, S.; Gaussier, E.; and Bouchard, G. 2016. Complex embeddings for simple link prediction. In Proceedings of the 33rd International Conference on Machine Learning, ICML'16.
Vashishth, S.; Joshi, R.; Prayaga, S. S.; Bhattacharyya, C.; and Talukdar, P. 2018. RESIDE: Improving distantly-supervised neural relation extraction using side information. In Proceedings of the 2018 Conference on Empirical Methods in Natural Language Processing, 1257-1266. Brussels, Belgium: Association for Computational Linguistics.

Vashishth, S.; Jain, P.; and Talukdar, P. 2018. CESI: Canonicalizing open knowledge bases using embeddings and side information. In Proceedings of the 2018 World Wide Web Conference, WWW '18, 1317-1327. Republic and Canton of Geneva, Switzerland: International World Wide Web Conferences Steering Committee.

Wang, Z.; Zhang, J.; Feng, J.; and Chen, Z. 2014a. Knowledge graph embedding by translating on hyperplanes. In Proceedings of the Twenty-Eighth AAAI Conference on Artificial Intelligence, AAAI'14, 1112-1119. AAAI Press.

Wang, Z.; Zhang, J.; Feng, J.; and Chen, Z. 2014b. Knowledge graph embedding by translating on hyperplanes. In Proceedings of the Twenty-Eighth AAAI Conference on Artificial Intelligence, AAAI'14, 1112-1119. AAAI Press.

Wang, Q.; Mao, Z.; Wang, B.; and Guo, L. 2017. Knowledge graph embedding: A survey of approaches and applications. IEEE Transactions on Knowledge and Data Engineering 29(12):2724 2743

Wang, T.-H.; Huang, H.-J.; Lin, J.-T.; Hu, C.-W.; Zeng, K.-H.; and Sun, M. 2018. Omnidirectional cnn for visual place recognition and navigation. arXiv preprint.

Xiao, H.; Huang, M.; and Zhu, X. 2016. Transg : A generative model for knowledge graph embedding. In Proceedings of the 54th Annual Meeting of the Association for Computational Linguistics, 2316-2325.

Yang, B.; Yih, W.; He, X.; Gao, J.; and Deng, L. 2014. Embedding entities and relations for learning and inference in knowledge bases. CoRR abs/1412.6575.

Zhang, F.; Yuan, N. J.; Lian, D.; Xie, X.; and Ma, W.-Y. 2016. Collaborative knowledge base embedding for recommender systems. In Proceedings of the 22nd International Conference on Knowledge Discovery and Data Mining, KDD '16, 353-362. 